\title{
Emprendimientos deportivos: Caso Subregión Sabana, Departamento deSucre, Colombia Sport entrepreneurship: Sub-region Sabana, Department of Sucre, Colombia
}

\author{
*José Ramón Sanabria Navarro, *Yahilina Silveira Pérez, **Lisbet Guillen Pereira, ***Digna Dionisia Pérez Bravo
}

*Corporación Universitaria del Caribe-CECAR (olombia), **Universidad Metropolitana del Ecuador (Ecuardor), ***Universidad Técnica Particular de Loja (Ecuardor)

\begin{abstract}
Resumen: El objetivo del estudio es analizar el estado actual de los emprendimientos deportivos de la subregión Sabana del Departamento de Sucre, Colombia. El año promedio de creación de los emprendimientos es 2016, administrados por hombres de 27- 56 años. Se estudiaron dos muestras, 900 clientes y 64 emprendedores deportivos. La metodología consiste en una investigación aplicada, con intervención en las comunidades, para lo cual se emplean métodos teóricos, empíricos y estadísticos. El diagnóstico inicial permite un análisis comparativo desde la percepción de clientes y emprendedores, a partir de las variables comportamiento, oferta y percepción sobre los emprendimientos deportivos. El procesamiento de datos es realizado mediante pruebas de análisis factorial para comprobar la unidimensionalidad de las variables, un análisis ANOVA para comprobar las hipótesis planteadas y un análisis regresión lineal de clientes para establecer el modelo de emprendimiento deportivo para la región estudiada. En los resultados se obtuvo que la calidad del servicio se relaciona lineal y positivamente con la eficiencia del servicio y el ambiente laboral, que existen diferencias entre la percepción del cliente y el emprendedor sobre la actividad física, en ese sentido, según el cliente percibe el servicio deportivo así es su comportamiento. Además, que la percepción que el emprendedor tienen sobre el servicio está condicionando su oferta y no las necesidades del cliente en la práctica de actividad física. El impacto de los resultados radica en el nuevo conocimiento que brinda para la sociedad en función de su actual aplicación desde la óptica del practicante. Palabras claves: emprendimiento deportivo, cliente, percepción, comportamiento, administración deportiva.
\end{abstract}

Abstract: The objective of the study is to analyze the current state of sporting enterprises in the Sabana subregion of the Department of Sucre, Colombia. Two samples were studies: 900 clients and 64 sports entrepreneurs. The average year of enterprise foundation is 2016 , run by males aged 27-56. The methodology consists of applied research, with intervention in the communities, for which theoretical, empirical and statistical methods are used. The initial diagnosis allows a comparative analysis based on the perception of clients and entrepreneurs, the behavior, supply and perception on sporting ventures. Data processing was performed through factorial tests to check the unidimensionality of the variables, an ANOVA analysis to check the hypotheses, and a linear regression analysis of clients to establish the model of sports entrepreneurship for the region studied. The results showed that the quality of the service is linearly and positively related to the efficiency of the service and the work environment demonstrating differences between the perception of the client and the entrepreneur about physical activity. In this sense, client's behavior as a result of the service quality. In addition, entrepreneur's perception about his services effects his offer; client's needs regarding PA are not considered. The impact of the results lies in the new knowledge it provides for society based on its current application from the perspective of the practitioner.

Key words: sports entrepreneurship, client, perception, behavior, sports administration.

\section{Introducción}

La práctica de deportes y el desarrollo de actividades físicas contribuyen al mantenimiento y mejora sustancial de la calidad de vida, la salud de las personas y la prevención de enfermedades no transmisibles, independientemente de la edad de los practicantes. Se considera al respecto que para que genere éxito este tipo de actividad y contribuir de manera acertada a los beneficios que aporta, debe ser planificada y dosificada, acorde a las particularidades de las variables fisiológicas, morfológicas, demográficas, sociólogas, entre otras (Tuero \& Marquez, 2010).

En este orden es importante resaltar que las sociedades modernas promueven con sus estructuras automatizadas el sedentarismo, relevando cada vez en mayor grado el esfuerzo físico. Existen ejemplos de esta inactividad física que pueden deberse a cuestiones no solo tecnológicas sino también de género (Sevil, Abós, Julian, Murillo \& GarcíaGonzález, 2015) y edad (Murillo, Julián, García-González, AlbarcaSos \& Zaragoza, 2014; Gil-Madrona, Pita-Lozano, Díaz-Suárez \& López-Sánchez, 2017), así como las creencias de los practicantes sobre sus habilidades en actividad física (Moreno-Murcia, Cervelló-Gimeno, Martínez-Galindo \& Moreno, 2013).

Cabe destacar que en diversas investigaciones científicas se ha podido avalar que la actividad física no solo es un abordaje teórico de la cultura física que analiza las condiciones fisiológicas y psicológicas del individuo, sino que se ha convertido también en un fenómeno social, político y económico que deviene en emprendimientos como parte integral de la gestión deportiva (Espejo \& Ezquerra, 2002; MoraRodríguez, 2009; Tuero \& Marquez, 2010; Acosta, 2012; González-

Fecha recepción: 03-04-18. Fecha de aceptación: 29-06-18

Lisbet Guillen Perein

guillenp7212@gmail.com
Montesinos \& Fernández-Santos, 2012; Abadia, 2014; Martínez, 2015; Arias, 2017).

En este orden Huertas, Valantine \& Crespo (2014) coinciden con Ratten \& Ratten (2011) al analizar el tema del emprendimiento en el ámbito deportivo, al respecto concuerdan con que el deporte es apreciado como un negocio que es emprendedor por naturaleza, debido a que este escenario cambia constantemente para adaptarse a las nuevas necesidades del negocio. En estepunto el deporte basado en el emprendimiento implica una actividad que es esencialmente innovadora, que está impulsada por una oportunidad y que tiene una visión orientada (Ratten, et al., 2011; Méndez, 2015).

Ratten (2012) precisa que el emprendimiento en el deporte se encuentra influenciado por un conjunto de valores que estimulan la intención de crear o desarrollar actividades con un carácter innovador en las organizaciones o en los individuos, y que por tanto se caracteriza por comportamientos de innovación, toma de riesgos y proactividad dentro del escenario deportivo.

Desde el punto de vista social, permite la inclusión de personas con todo tipo de condiciones, estatus y niveles sociales preestablecidos por las distintas sociedades. En este orden de análisis, Norbert Elias, reconocido sociólogo, planteó en el 1939, sostiene que la mejor forma de conocer una sociedad es a través del conocimiento que se tienen del deporte, en otras palabras, si se es capaz de saber cómo se práctica el deporte, qué importancia tiene este en la vida de las personas, qué programas existen, competiciones y demás componentes del sistema cognitivo, entonces es posible caracterizar la sociedad desde el deporte (Urteaga, 2013); en este orden se asume la idea que no es posible desconocer el alto compromiso social que posee el deporte en relación a la inclusión (Abadia, Medina, Sánchez, Sánchez, Bantulà \& Morejón, 2016; Guillen, Arma \& Formoso, 2018).

Como fenómeno económico, la actividad física y los deportes se han convertido en una idea de negocio con el surgimiento de diferentes tipos de emprendimientos que transitan desde gimnasios de pequeña, 
mediana y sofisticada estructura, empresas de fitness, clubes deportivos con altos índices de ingresos, patrocinios de marcas, entre otros (Puigarnau, Camerino, Castañer, Prat \& Anguera, 2016; Guillen, Martinez \& Montano, 2018).

Siguiendo estas ideas de pensamiento podría plantearse el supuesto de que es el ser social es capaz de influir en la evolución de la actividad física y los deportes, de forma integrada desde estas tres perspectivas, pues como miembro de la sociedad, puede incidir y proponer políticas públicas en función del deporte y crea nuevas empresas con este tipo de concepto (Guillen, et al, 2018).

Para comprender el valor del emprendimiento en el senario del deporte es preciso recurrir al concepto cuya introducción es realizada por Richard Cantillon en el 1755 según Stevenson \& Jarillo (1983) y Rodríguez (2009); este autor enfoca el concepto hacia la actividad económica, sin embargo, se valora el posicionamiento de Jean-BaptisteSay al analizar el concepto desde la dimensión de la organización de factores de producción (Suárez \& Vásquez, 2015).

Por su parte Alfred Marshall en el 1919, al analizar la teoría económica del empresario identifica al emprendedor como líder por naturaleza, dispuesto a actuar bajo las condiciones de incertidumbre que causa la ausencia de información completa, al respecto Peter Drucker (1985) sostiene que el emprendedor es un empresario innovador y Gifford Pinchot (1985) los expone como personas que sueñan y logran hacer realidad lo que imaginan pues poseen un espíritu empresarial según Formichella (2004), mientras que Schumpeter citado por Herrera(2014) lo clasifica como fundador de una nueva empresa, un innovador que rompe con la forma tradicional de hacer las cosas, con las rutinas establecidas y otras conceptualizaciones son agente económico que une todos los medios de, carácter emprendedor asociado al cambio social y sentido material, necesidades sociales y económicas (Holcombe, 1998); por lo tanto, un profesional del deporte puede ser un emprendedor deportivo.

Al respecto, en Colombia, la actividad física y el deporte han cobrado importancia en los últimos años debido a que el gobierno ha decidido convertir el deporte en uno de sus estandartes. Existen programas nacionales como «Hábitos y estilos de vida saludables» del Departamento Administrativo del Deporte, la Recreación, la Actividad Física y e Aprovechamiento del Tiempo Libre (COLDEPORTES), de los cuales se beneficia un gran porcentaje de la población.

De igual forma se han construido instalaciones, estructuras y escenarios deportivos con altos estándares de calidad que van en pro del desarrollo del deporte. Independientemente de estos esfuerzos, según Kelley, Singer \& Herrington (2016) el 70\% de la población adulta percibe el emprendimiento deportivo como una carrera profesional, sin embargo, solo el $5 \%$ logra emprender.

Obsérvese que la idea de emprender surge debido a su viabilidad y acogida por parte del mercado y su posicionamiento; como resultado el emprendimiento se ha convertido en una fuente importante de ingreso. En el Departamento de Sucre los emprendedores deportivos son personas que debido a las pocas oportunidades de empleo que la sociedad ofrece han incursionado en este mundo empresarial.

Podría decirse que el campo del deporte ha crecido solo en las principales ciudades y no en las regiones rurales del país, ni a nivel de municipios y ni en las comunidades. Por tanto, resulta un desafío para el campo de la administración deportiva, determinar el comportamiento e interrelación de las variables que conducen a un profesional del deporte a crear y mantener un emprendimiento deportivo en comunidades rurales.

$\mathrm{Al}$ respecto existen estudios que demuestran la relación entre la formación del emprendedor, su intención de emprender y motivaciones (García \& Pires, 2010; Barba-Sánchez \& Atienza-Sahuquillo, 2018), en este orden los emprendedores deportivos de Sucre no poseen los conocimientos básicos para desarrollar la actividad física, basándose en el empirismo, por tanto, es evidente que el cliente juega un papel primordial en el desarrollo y evolución de los emprendimientos.

En relación con el tema Rial, Varela, Rial \& Real (2010), plantean que la orientación hacia el cliente ha modificado los servicios deportivos, reflejado en el incremento de gimnasios y centros de deporte. Por lo que, no solo el emprendedor es un actor importante en este sistema sino también el cliente, su comportamiento, la visión que tiene del negocio, necesidades, deseos y por ende la demanda del servicio deportivo. En concordancia el practicante de deportes, como cliente potencial, es apreciado como uno de los actores principales de un emprendimiento deportivo, por lo tanto, resulta imprescindible identificar sus preferencias y necesidades al momento de realizar actividades físicas y deportes.

Basado en esto planteamos que objetivo de la investigación es analizar el estado actual de los emprendimientos deportivos de la subregión Sabana del Departamento de Sucre, Colombia. Teniendo como objetivos específicos: 1. Caracterizar el emprendimiento deportivo en base al análisis comparativo entre la percepción del cliente y la percepción de los emprendedores del servicio deportivo; 2. Analizar el comportamiento del cliente y la oferta del servicio deportivo; 3. Proponer un modelo que refleje el comportamiento de los emprendimientos deportivos en relación a los resultados obtenidos.

\section{Metodología}

La estructura de la investigación partió de la identificación de los elementos teóricos-metodológicos del emprendimiento deportivo. En un segundo momento, se centró en determinar la percepción de los clientes y emprendedores del servicio deportivo en la subregión Sabana del Departamento de Sucre. Esto aportó los referentes básicos que permitieron la determinación de los elementos y componentes del modelo teórico del emprendimiento deportivo, el cual fue validado a través de las hipótesis establecidas. Por tanto, fue necesario comprobar si existe relación entre la percepción y comportamiento que tienen el cliente sobre el emprendimiento deportivo, así como compararlos con la percepción de los emprendedores y la oferta que ofrecen.

La metodología empleada asume el método dialéctico materialista en cuanto a su historicidad, multidimensionalidad, funcionalidad y su carácter sistémico. En relación se da respuesta a cada uno de los objetivos planteados. Se emplearon diferentes métodos y/o técnicas del nivel teórico, empírico y estadístico matemático en cada una de las fases de investigación declaradas: Diagnóstico, Elaboración y Validación; dentro de estos se encuentran el método inductivo- deductivo, análisis y síntesis, el método histórico-lógico utilizado para el establecimiento de las teorías objeto de estudio, el análisis bibliográfico, la revisión de documentos y la encuesta utilizada para identificar elementos relevantes del estado actual del objeto de estudio. De igual manera se empleó la modelación que como método permitió la representación teórica de la realidad de forma gráfica, el método sistémico estructural funcional con el objetivo de ajustar el funcionamiento del modelo como sistema, la triangulación metodológica utilizada para contrastar los datos desde diferentes ángulos, la técnica de distribución empírica de frecuencia requerida para el análisis descriptivos de los datos consignados, la medición y el método de Criterio de Experto, el estudio estuvo condicionada a la comprobación de las siguientes hipótesis de investigación:

$\mathrm{H}_{1}$ : La diferencia entre la percepción del cliente y la del emprendedor del servicio deportivo caracteriza el emprendimiento.
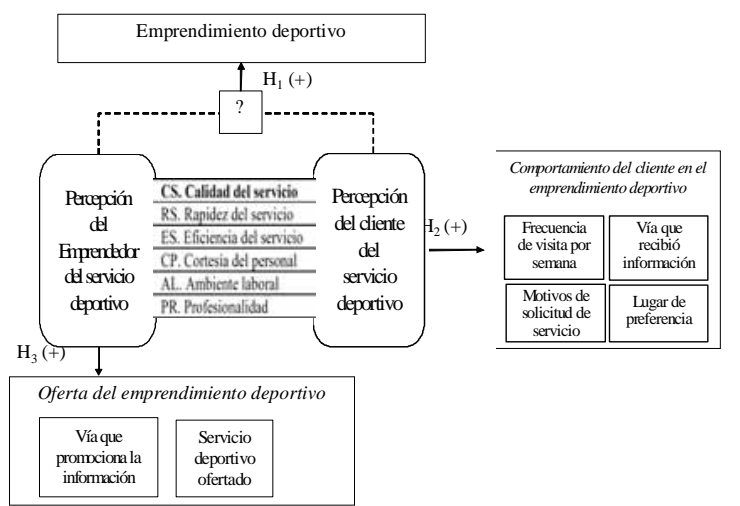

Figura 1. Modelo teórico de la investigación. 
$\mathrm{H}_{2}$ : La percepción del cliente del servicio deportivo influye en su comportamiento en el emprendimiento deportivo.

$\mathrm{H}_{3}$ : La percepción del emprendedor de servicio deportivo influye en la oferta del emprendimiento deportivo.

Empleando para ello el análisis factoriales, análisis deANOVAy la regresión lineal. El modelo que se presenta en la figura (1), establece la relación de las hipótesis de investigación en un orden lógico para la intervención. Tiene en cuenta el cómo se deben integrar cada una de las variables para que se logren satisfacer las necesidades de los clientes. Posee un orden cíclico para garantizar una retroalimentación constante en el proceso para corregir futuros errores que se puedan ir presentando en la puesta en marcha.

\section{Diseño de las muestras}

En correspondencia con lo planteado se parte de la representación teórica de la realidad de forma gráfica y se procede al diseño de un modelo teórico. Para ello se trabajó con 64 emprendimientos de los municipios dela subregión Sabana del Departamento de Sucre: Sampués, Corozal, Los Palmitos, San Pedro, Buenavista, Sincé, Betulia, Galeras y El Roble. En el caso del desglose de emprendimientos por municipios el mayor porcentaje de emprendimientos fue ubicado en Corozal con un 45.3\% (29 municipios) (Tabla 1).

\begin{tabular}{|c|c|c|c|c|}
\hline & Frecuencia & Porcentaje & Porcentaje válido & Porcentaje acumulado \\
\hline Sincé & 7 & 10.9 & 10.9 & 10.9 \\
\hline Betulia & 3 & 4.7 & 4.7 & 15.6 \\
\hline El Roble & 1 & 1.6 & 1.6 & 17.2 \\
\hline Sampués & 10 & 15.6 & 15.6 & 32.8 \\
\hline Buenavista & 2 & 3.1 & 3.1 & 35.9 \\
\hline Galera & 5 & 7.8 & 7.8 & 43.8 \\
\hline San Pedro & 4 & 6.3 & 6.3 & 50.0 \\
\hline Los Palmitos & 3 & 4.7 & 4.7 & 54.7 \\
\hline Corozal & 29 & 45.3 & 45.3 & 100.0 \\
\hline Total & 64 & 100.0 & 100.0 & \\
\hline
\end{tabular}

\section{Muestra 2. Clientes}

Los nueve municipios declarados aportaron a la investigación un total de 900 clientes, para cuya selección se empleó un diseño muestral no probabilístico por cuotas asignando 100 cuotas a cada municipio, la selección de clientes a encuestar se realizó mediante un muestreo no probabilístico por criterios. Se consideróla disposición de colaborar en la investigación, que fueran clientes de los emprendimientos deportivos y tener tres meses continuos asistiendo a alguno de los emprendimientos de la subregión.

La caracterización de la muestra de clientes (Tabla 2) se realizó a partir de las variables demográficas: edad (16-26 años $\pm 41 \%$; 27-45 años $\pm 45 \%$; $46-60$ años $\pm 16 \%$ ), sexo (hombres $=89 \%$, mujeres=11\%) y profesión (empleos informales $=40 \%$, profesionales $=33 \%$, estudiantes $=21 \%$, técnicos $=5 \%$ ), indica que los clientes poseen una edad adecuada para la realización de ejercicios físicos de media y alta intensidad, el registro de datos devela la baja asistencia de sujetos con más de 60 años.

\begin{tabular}{|c|c|c|c|c|c|}
\hline \multirow{2}{*}{$\begin{array}{c}\text { Variables } \\
\text { demográficas }\end{array}$} & \multirow{2}{*}{ Escala } & \multicolumn{2}{|c|}{ Muestra 1: Clientes } & \multicolumn{2}{|c|}{ Muestra 2: Emprendedores } \\
\hline & & Frecuencia & Porcentaje válido & Frecuencia & Porcentaje válido \\
\hline \multirow{4}{*}{ Edad } & $16-26$ & 371 & 41.2 & 13 & 20.3 \\
\hline & $27-45$ & 402 & 44.7 & 33 & 51.6 \\
\hline & $46-60$ & 121 & 13.4 & 17 & 26.6 \\
\hline & +60 & 6 & 0.7 & 1 & 1.6 \\
\hline \multirow{2}{*}{ Sexo } & Femenino & 101 & 11.2 & 5 & 7.8 \\
\hline & Masculino & 799 & 88.7 & 59 & 92.2 \\
\hline \multirow{4}{*}{ Profesión } & Profesional & 301 & 33.4 & 23 & 35.9 \\
\hline & Técnico & 47 & 5.2 & 5 & 7.8 \\
\hline & Estudiante & 188 & 20.9 & 3 & 4.7 \\
\hline & Empleo Informal & 364 & 40.4 & 33 & 51.6 \\
\hline & otal & & 900 & & 64 \\
\hline
\end{tabular}

En correspondencia se trabajó por ende con 64 emprendedores deportivos, los que representan el 100\% de la población de técnicos que asisten directamente al cliente. La caracterización de la población correspondiente a emprendedores develó que el porcentaje mayor de edad (51.6\%) se concentró fundamentalmente en el rango de 27 a 45 años, el 92.2\% pertenecen al sexo masculino, el 51.6\% (33 técnicos) no tienen profesión formal definida y el 35.9\% (23 casos) son profesionales (Tabla 2).

\section{Procedimiento, instrumentos y variables del estudio}

Para el desarrollo de la investigación, un elemento importante fue la identificación de las variables, las cuales pueden asumir valores y características diferentes (Dancey \& Reidy, 2006). El cuestionario estructurado como documento escrito utilizado en la encuesta a clientes, incluyó preguntas relativas a las variables: comportamiento del cliente y percepción de los emprendimientos deportivos (Tabla 3).

El cuestionario estructurado utilizado en la encuesta a emprendedores incluyó la oferta y percepción del emprendedor del servicio deportivo (Tabla 4).

Para las variables V1. Comportamiento de los clientes del servicio en los emprendimientos deportivos y E1. Oferta del emprendimiento deportivo, se aplicó una escala nominal que identifica numéricamente la escala verbal en las posibles respuestas a seleccionar por los clientes encuestados.

La escala para las variables V2. Percepción del cliente del servicio en los emprendimientos deportivos y E2. Percepción del emprendedor del servicio deportivo, está determinada en una escala Likert con medición ordinal a los cuales se le otorgó un peso numérico que va desde 1=Pésimo, 2= Muy malo, 3= Malo, 4= Regular, 5=Bueno, 6=Muy bueno y 7=Excelente (Fischer \& Navarro, 1999), se asume el procedimiento por el hecho de la alta confiabilidad obtenida en estudios anteriores (Pullés, Gutiérrez \& Llorens, 2013), contrastada con una escala multi-itens (Bojica, Fuentes \& Ruiz, 2012).

En relación con las subvariables en la percepción del cliente y percepción del emprendedor, la calidad del servicio es una de las que mayor efecto tiene en los clientes. En los estudios empíricos de Rial et al., (2010), la calidad percibida por los clientes se divide en dos dimensiones, personal e instalaciones. Ascenso-Campos, Da Costa-Simões, \& Alves-Franco (2017), por su parte se refiere a la calidad del entrenador en cuatro dimensiones calidad profesional, calidad relacional, calidad técnica y calidad pedagógica. Es en este sentido que los emprendimientos deportivos no pueden descuidar los criterios asociados a la gestión de la calidad que agregan valor a su servicio. La atención 
al cliente, el trato y la forma de lidiar con ellos, es una de las cuestiones que no deben desatender en los emprendimientos deportivos (Rial, et al., 2010). Pero no solo debe tenerse en cuenta este criterio hacia el exterior sino también entre el colectivo laboral, pues los clientes suelen percibir si existen problemas en el ambiente laboral (Puigarnau, et al., 2016). El personal debe estar bien formado y ser profesionales (Rial, et al., 2010). El objetivo de este instrumento es realizar una comparación de la información entre clientes y emprendedores.

La escala que se propone es validada según la fiabilidad utilizada en el procesamiento estadístico con la técnica SPSS 21.0. Uno de los criterios que se tuvo en cuenta en el inicio y el desarrollo de la investigación es el criterio de la subjetividad, que puede en investigaciones de naturaleza social limitar el resultado de percepción de los emprendedores que conforman la muestra (Xavier, Martins \& Lima, 2008).

\section{Resultados}

\section{Análisis de confiabilidad de la escala y estadística descriptiva}

Las muestras de 900 clientes de los prendimientos deportivos arrojaron un $100 \%$ de encuestas válidas. ElAlfa de Cronbach obtenido permite comprobar la fiabilidad de las escalas de las variables para clientes de .919. Por su parte los 64 emprendedores obtuvieron una fiabilidad igual a .784. En ambos casos los valores se consideraron válidos para su procesamiento (Tabla 5).

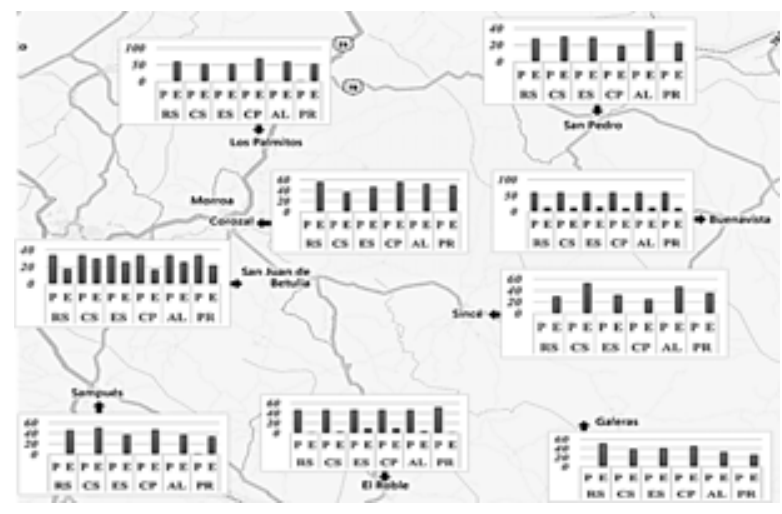

Figura 3. Tabulación cruzada de percepción del cliente del servicio deportivo por municipio $\mathrm{P}=$ Pésimo; E= Excelente; $\mathrm{RS}=$ Rapidez del servicio; $\mathrm{CS}=$ Calidad del servicio; ES=Eficiencia

del servicio; $\mathrm{CP}=$ Cortesía del personal; $\mathrm{AL}=$ Ambiente laboral; $\mathrm{PR}=$ Profesionalidad

El análisis de la tabulación cruzada de la frecuencia de visita de clientes a los emprendimientos deportivos refleja que el comportamiento los mismos es de una frecuencia de visita de tres veces por semana lo hacen fundamentalmente por el motivo de recibir un mejor servicio, seguido de la calidad, recibieron la información en la comunicación cara a cara y prefieren los locales deportivos para realizar las actividades físicas (Tabla 6).

La ventaja de que los clientes se hayan enterado de la oferta de actividad física por el método cara a cara es que los entrenadores o personal del emprendimiento ha logrado conversar con el cliente, conocer sus condiciones físicas y explicarle que tipo de servicio es el más recomendable, frecuencia entro otras cuestiones relativas a la oferta que brinda. La diferencia con recibir la información directamente en el local es cuando esta se limita exclusivamente a ofrecer una comunicación general que no es necesariamente personalizada. Los clientes que asisten dos veces que es el otro grupo significativo, lo hace considerando la calidad de la actividad física que se oferta, prefiriendo también los locales deportivos.

\begin{tabular}{cccccc} 
& CS. Calidad del servicio & 6.41 & .66 & .436 & .775 \\
Emprendedor & RS. Rapidez del servicio & 6.31 & .871 & .758 & .778 \\
& E2. Percepción del emprendedor del ES. Eficiencia del servicio & 6.41 & .786 & .618 & .782 \\
servicio deportivo & C. Cortesía del personal & 6.28 & .734 & .539 & .781 \\
& AL. Ambiente laboral & 6.52 & .734 & .539 & .777 \\
& PR. Profesionalidad & 6.52 & .724 & .524 & .776 \\
\hline
\end{tabular}

Por su parte en la figura (2) se observa una discrepancia entre la percepción que sobre el servicio tienen tanto los clientes como los emprendedores demostrando el desconocimiento que existe en los emprendimientos deportivos sobre la aceptación de sus servicios en el mercado, principalmente en cuanto a la profesionalidad.

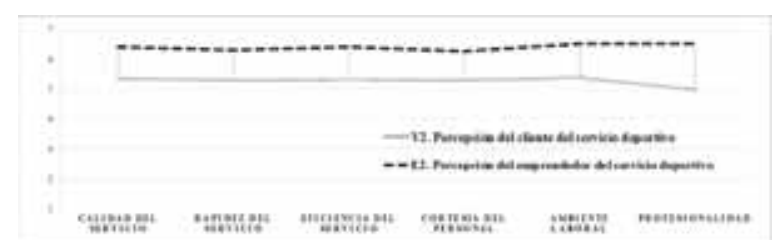

Figura 2. Perfil comparativo entre las percepciones del servicio deportivo de clientes y emprendedores

Significa que los emprendedores están sobrevaluando sus servicios, superior a la percepción que el cliente tiene sobre lo que recibe. Esto podría estar conduciendo a una insatisfacción del cliente, esto permite comprobar $\mathrm{H}_{1}$

En la tabulación cruzada de la percepción del cliente (figura 3) Buenavista es el municipio con una oferta deportiva calificada de pésima, seguido de Betulia y El Roble. Mientras tanto, Los Palmitos y Corozal ofrecen un servicio entre bueno y excelente, según lo percibe el cliente.

$\mathrm{P}=$ Pésimo; $\mathrm{E}=$ Excelente; $\mathrm{RS}=$ Rapidez del servicio; $\mathrm{CS}=\mathrm{Calidad}$ del servicio; $\mathrm{ES}=$ Eficiencia del servicio; $\mathrm{CP}=$ Cortesía del personal; $\mathrm{AL}=$ Ambiente laboral; $\mathrm{PR}=$ Profesionalidad
Tabla 6

Tabulación cruzada por frecuencia de visita del cliente al emprendimiento deportivo vs. Comportamiento del cliente en el emprendimiento deportivo

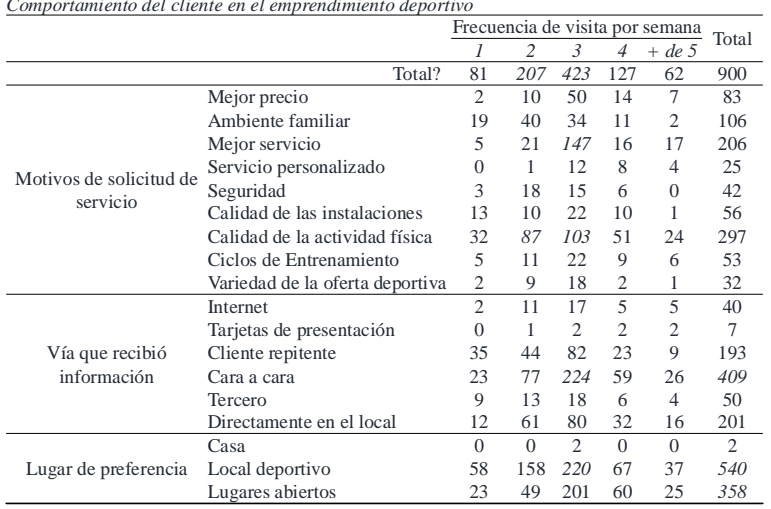

Tabla 7

Tabulación cruzada por frecuencia de Servicios deportivos ofertados vs. Oferta del mprendimiento deportivo

\begin{tabular}{clccccc}
\hline & & $\begin{array}{c}\text { Entrenamiento } \\
\text { deportivo }\end{array}$ & Recreación & \multicolumn{2}{c}{$\begin{array}{c}\text { Actividad } \\
\text { física y salud }\end{array}$} & Total \\
\hline & Total? & 53 & 3 & 8 & 64 \\
\hline \multirow{4}{*}{ Promoción } & Internet & 12 & 1 & 2 & 15 \\
de la & Tarjetas de presentación & 12 & 1 & 0 & 13 \\
información & Cliente repitente & 8 & 0 & 0 & 8 \\
& Cara a cara & 6 & 0 & 3 & 9 \\
& Tercero & 9 & 0 & 3 & 12 \\
& Directamente en el local & 6 & 1 & 0 & 7 \\
\hline
\end{tabular}


Realizando la tabulación cruzada de la variableE1, el análisis de la oferta del emprendimiento deportivo (Tabla 7), indica que el medio que más utilizan para la promoción de la oferta son Internet y las Tarjetas de presentación. Esto contradice la forma más frecuente utilizada por los clientes, comprobando $\mathrm{H}_{3}$.

\section{Análisis factorial}

El primer paso ha sido determinar la Medida Kaiser-Meyer-Olkin $>$.8, que en conjunto con $\mathrm{p}=.0000$, indican que es posible aplicar el análisis indicado (Tabla 8), con una varianza total explicada en un 92.5\% y $54.68 \%$, respectivamente, con cargas factoriales superiores a .5.

En este caso se demuestra que todas las variables que cualifican la valoración del servicio de emprendimiento deportivo según la percepción del cliente generan un único factor y lo mismo sucede en la percepción de los emprendedores. Por tanto, el análisis factorial en ambas muestras permite comprobar la unidimensionalidad de las variables V2. Percepción del cliente del servicio deportivo y E2. Percepción del emprendedor del servicio deportivo.

\section{Análisis de ANOVA}

Para contrastar las hipótesis se realiza un análisis ANOVAdonde se demuestra que la percepción del cliente del servicio de emprendimiento deportivo no influye en su lugar de preferencia para realizar actividades físicas; sin embargo, si lo hace en el resto de las variables (Tabla 9). En cuanto a su comportamiento de incrementar la frecuencia con que visita por semana, tienen un mayor énfasis el profesionalismo, rapidez del servicio y cortesía del personal. Tanto en la vía en que recibe la información como los motivos de su visita, la percepción del cliente influye positivamente. Por lo tanto, se cumple parcialmente hipótesis $\mathrm{H}_{2}$

\section{Análisis Regresión Lineal de clientes}

Habiendo demostrado en los resultados anteriores, la importancia de la variable percepción del cliente del servicio deportivo, es primordial determinar cuál es el modelo que siguen las dimensiones de esta, ubicando a la calidad del servicio como variable dependiente. El análisis de regresión lineal permite establecer el modelo de emprendimiento deportivo para la región estudiada desde la percepción del cliente. Utilizando el método de por pasos (Criterios: Probabilidad-de-F-para-entrar <=

\begin{tabular}{|c|c|c|c|}
\hline & & V2. & $E 2$. \\
\hline Medida Kaiser-Meyer-O & in de adecuación de muestreo & .951 & 0.781 \\
\hline \multirow{3}{*}{$\begin{array}{c}\text { Prueba de esfericidad de } \\
\text { Bartlett }\end{array}$} & $\begin{array}{l}\text { Aprox. Chi-cuadrado } \\
\end{array}$ & 9519.873 & 134.737 \\
\hline & $\mathrm{gl}$ & 15 & 15 \\
\hline & $\begin{array}{l}\text { Sig. } \\
\end{array}$ & .000 & .000 \\
\hline & Varianza Total explicada (\%) & 92.462 & 54.682 \\
\hline \multicolumn{2}{|c|}{ Componente } & $\begin{array}{c}\text { Cargas } \\
\text { Factoriales }\end{array}$ & $\begin{array}{c}\text { Cargas } \\
\text { Factoriales }\end{array}$ \\
\hline \multicolumn{2}{|l|}{ CS. Calidad del servicio } & .972 & .822 \\
\hline \multicolumn{2}{|l|}{ ES. Eficiencia del servicio } & .972 & .805 \\
\hline \multicolumn{2}{|l|}{ AL. Ambiente laboral } & .969 & .732 \\
\hline \multicolumn{2}{|l|}{ CP. Cortesía del personal } & .963 & .728 \\
\hline \multicolumn{2}{|l|}{ RS. Rapidez del servicio } & .960 & .710 \\
\hline \multicolumn{2}{|l|}{ PR. Profesionalidad } & .932 & .623 \\
\hline
\end{tabular}

Tabla 9.

ANOVA del Comportamiento del cliente en el emprendimiento deportivo

V2 Percepción del cliente V1. Comportamiento del cliente en el emprendimiento deportivo Frecuencia de Vía que recibió Motivos de solicitud Lugar de

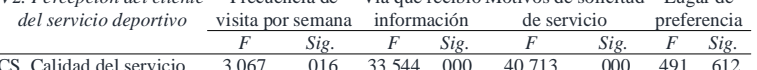
\begin{tabular}{lllllllll}
\hline CS. Calidad del servicio & 3.067 & .016 & 33.544 & .000 & 40.713 & .000 & .491 & .612 \\
RS. Rapidez del servicio & 3.997 & .003 & 29.676 & .000 & 50.410 & .000 & .720 & .487
\end{tabular} $\begin{array}{llllllllll}\text { ES. Eficiencia del servicio } & 3.104 & .015 & 35.737 & .000 & 45.154 & .000 & .692 & .501\end{array}$ $\begin{array}{llllllllll}\text { CP. Cortesía del personal } & 3.606 & .006 & 33.846 & .000 & 50.964 & .000 & 1.125 & .32\end{array}$ \begin{tabular}{lllllllll} 
AL. Ambiente laboral & 2.816 & .024 & 36.676 & .000 & 50.651 & .000 & .724 & .485 \\
PR. Profesionalidad & 6.012 & .000 & 20.423 & .000 & 35.938 & .000 & 1.330 & .265 \\
\hline
\end{tabular} Tabla 10.

\begin{tabular}{ccccc}
\multicolumn{2}{l}{ Resumen del modelo } \\
\hline Modelo & $R$ & $R$ cuadrado & R cuadrado ajustado & Error estándar de la estimación \\
\hline 1 & $.938_{\mathrm{a}}$ & .879 & .879 & .711 \\
2 & $.953_{\mathrm{b}}$ & .908 & .908 & .620 \\
3 & $.95 \mathrm{~b}_{\mathrm{c}}$ & .917 & .917 & .591 \\
4 & $.96 \mathrm{~d}_{\mathrm{d}}$ & .921 & .921 & .577 \\
5 & $.966 \mathrm{e}_{\mathrm{e}}$ & .922 & .922 & .572 \\
\hline a. Predictores: (Constante), Eficiencia del servicio
\end{tabular}

b. Predictores: (Constante), Eficiencia del servicio, Cortesía del personal

b. Predictores: (Constante), Eficiencia del servicio, Cortesía del personal
c. Predictores: (Constante), Eficiencia del servicio, Cortesía del personal, Rapidez del servicio

d. Predictores: (Constante), Eficiencia del servicio, Cortesía del personal, Rapidez del servicio, d. Predictores: (Constion
Ambiente Laboral

e. Predictores: (Constante), Eficiencia del servicio, Cortesía del personal, Rapidez del servicio, e. Predictores: (Constante), Eficien

$\mathrm{f}$. Variable dependiente: Calidad del Servicio
.050 , Probabilidad-de-F-para-eliminar $>=.100)$, se obtuvieron dos modelos aceptables: Modelo (1) y Modelo (2) (Tabla 10).

La ANOVA (Tabla 11) informa que existe relación significativa entre las variables, pues el nivel crítico (Sig.) igual a cero, indica que las variables están linealmente relacionadas.

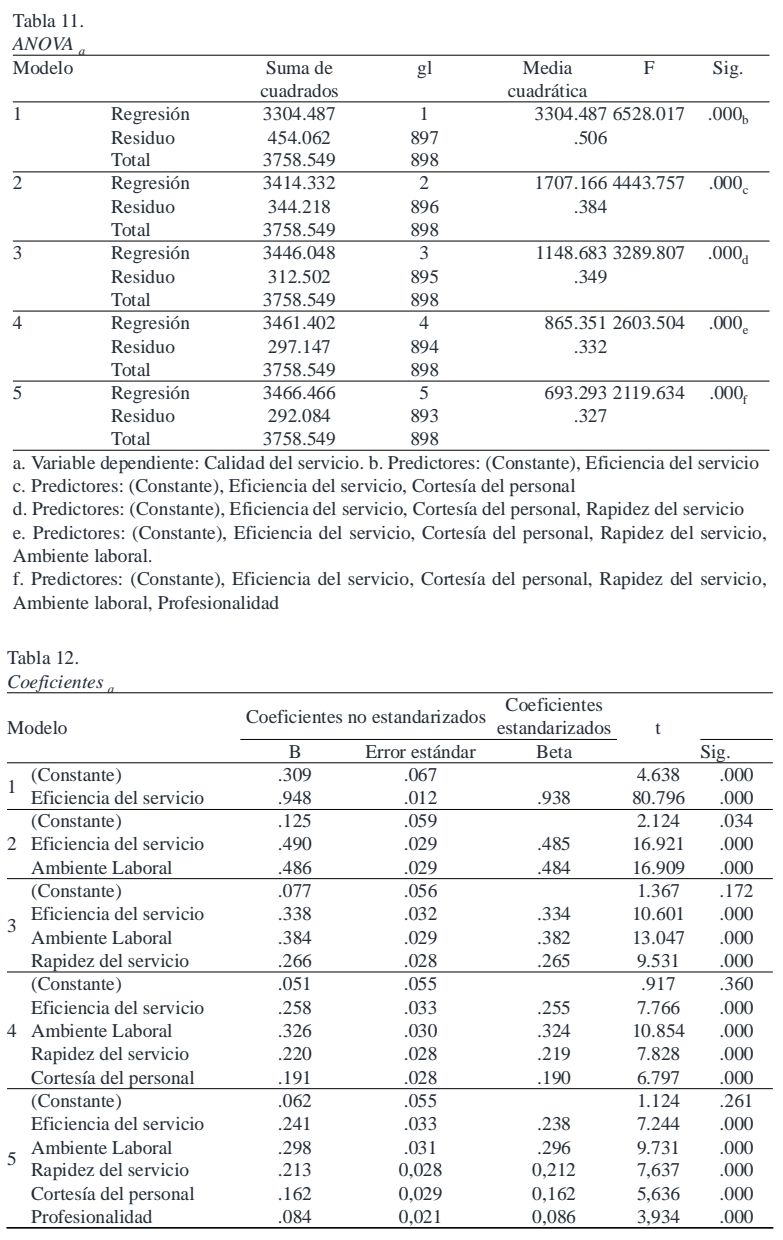

a. Variable dependiente: Calidad del Servicio

Del análisis de esta, se obtuvo que las variables que entraron al modelo fueron Calidad del servicio (CS) como dependiente, mientras que Eficiencia del servicio(ES) y Ambiente laboral (AL) como independientes:

Modelo 1: CS= .309+.948*ES (1)

Interpretación: La calidad del servicio se relaciona linealmente con la eficiencia del servicio de manera positiva. El coeficiente de la constante muestra que para cada puntuación de la eficiencia del servicio la calidad aumentará en .309 puntos. $\mathrm{H}_{\mathrm{I}(2)}$ se cumple parcialmente pues no todas las variables entraron en el modelo.

Modelo 2: CS = .125 + .490* ES+ .486*AL (2)

Interpretación: La calidad del servicio se relaciona linealmente con la eficiencia del servicio y el ambiente laboral de manera positiva, en un coeficiente de 125 puntos. El resto de los modelos tienen una significación mayor a .05, lo que indica que no son estadísticamente significativos por tanto no son tomados en cuenta.

\section{Discusión}

El presente trabajo de investigación tuvo como objetivo analizar el estado actual de los emprendimientos deportivos de la subregión Sabana del Departamento de Sucre, Colombia. Teniendo en cuenta el análisis de las teorías, los resultados de las evaluaciones de los miembros de la muestra y la validación de las hipótesis del modelo podemos plantear que se han cumplido los objetivos trazados.

Uno de los primeros resultados obtenidos en el estudio obedece a la poca oferta de actividades físicas adaptadas a las particularidades y 
posibilidades de las personas adultas mayores en estos establecimientos, así como las condiciones precarias para la inclusión social. Esto constituiría una pérdida de visión de los emprendimientos deportivos, pues en los últimos años, este segmento constituye uno de los de mayor crecimiento e importancia, sobre todo en América Latina (Martínez del Castillo, Jiménez-Beatty, Santacruz, Martín \& Rivero, 2011; Acosta, 2012).

Por otra parte, los hombres son los que más asisten a estos establecimientos algo que se contradice con estudios previos que demuestran que el sexo femenino es más propenso a tener una actividad deportiva más diversa (Alvariñas-Villaverde, López-Villar, Fernández-Villarino, \&Alvarez-Esteban, 2017). Esta variedad podría sugerirle a los emprendedores que amplíen sus ofertas orientadas tanto a circunscribir personas con discapacidad, necesidades de inclusión social, como a las mujeres.

El análisis derivado de los resultados permite confirmar que la diferencia entre la percepción del cliente y del emprendedor del servicio deportivo caracteriza el emprendimiento. Utilizando como referente el estudio de Fernández-Villarino, González-Valeiro, Toja-Reboredo, \& Carreiro da Costa (2017), que comprueba de que cuando se tiene una actitud positiva hacia la educación física esta es correspondiente con un incremento en la práctica de la actividad física, por tanto traspalando este resultado a la investigación actual, significaría que tener un conocimiento previo sobre la práctica del deporte conduciría a las personas a realizar con mayor frecuencia la actividad física. Sin embargo, en la frecuencia en que visita el centro, incide de forma significativa indicadores relacionados con el profesionalismo, la rapidez del servicio y la cortesía del personal.

Otro orden del análisis, en los resultados obtenidos se verifica parcialmente que la calidad del servicio percibida por el cliente influye en su lugar de preferencia para realizar actividades físicas donde se aprecia una inclinación hacia lugares abiertos y locales deportivos, por lo que al relacionarlo con el precedente anterior no basta con el conocimiento, sino que el lugar para realizarlo es igual de importante. Tal es el caso de la propuesta desarrollada por Lamoneda-Prieto, \& HuertasDelgado (2017), quienes demuestran la importancia de los espacios interiores, lo que sería para nosotros los locales deportivos, para que las mujeres realicen la actividad física. En este sentido el 60\% de los clientes manifiestan preferir realizar la actividad física en locales deportivos, más que en espacios abiertos o en casa.

Los emprendimientos deportivos con mayor afluencia son los gimnasios y según estudios contemporáneos, una de las principales problemáticas presentes en estos establecimientos es la ausencia de entrenadores o preparadores físicos (Castro-López, Cachón, Valdivia-Moral, \& Zagalaz, 2015). Específicamente en la muestra estudiada de 64 emprendedores deportivos, el 51.6\% no poseen una formación en deportes ni en ciencias de la cultura física, lo cual puede que este reflejado en el resultado de que la variable profesionalidad haya sido excluida en el modelo de regresión lineal.

Diversos estudios confirman que la calidad percibida constituye un constructo enormemente controvertido y complejo, pero resulta clave en los modelos de gestión deportiva; el estado del indicador ofrecer a los profesionales y directores de los centros deportivos elementos relevantes del servicio para su perfeccionamiento (Rial, et al., 2010; Morales \& Gálvez, 2011; Ramirez, Lorenzo \& López, 2016; Nuviala, Tamayo-Fajardo, Ruiz-Alejos, Nuviala \& Dalmau, 2017).

De similar forma García, Bernal, Lara \& Galán (2013) demuestran mediante un estudio la relación entre la calidad percibida de servicio deportivo y la fidelidad del cliente utilizando el método de regresión lineal, los resultados presentados por los autores aportaron un conocimiento decisorio para la gestión de las instalaciones deportivas, planteándose las bases para la comprensión de estos constructos en la industria del fitness, lo que refuerza la importancia del análisis realizado en esta investigación.

Con respecto a la identificación de los elementos teóricos y metodológicos del emprendimiento deportivo se evidenció que este está determinado por las variables Eficiencia del servicio, Ambiente laboral, Rapidez del servicio, Cortesía del personal, Profesionalidad y Calidad del servicio, lo que contrasta con estudios previos que se centran en similar evaluación (Rial, et al., 2010; Nuviala, Teva-Villén, PérezOrdás, Grao-Cruces, Tamayo \& Nuviala, 2014; Nuviala, et al., 2017)

Contrasta con los datos expuesto el aporte de Nuviala, et al. (2017) los autores demuestran la validez y fiabilidad de un instrumento que permite determinar la calidad, valor y satisfacción de usuarios deportivos; desde la teoría justifican la importan del estudio y el redimensionamiento de la variable como resultado del desarrollo percibido en la dinámica del emprendimiento deportivo y la aceptación por parte de los usuarios que realizan actividades no dirigidas en diferentes organizaciones deportivas.

El análisis de la variable demográfica develó un comportamiento hacia el incremento de la frecuencia con que se visitan los centros deportivos, dentro de este se expone el comportamiento de la muestra de forma segmentada; la dinámica del estudio apuntó hacia la necesidad de evaluar la variable Motivos de solicitud del servicio. Su comportamiento preponderante hacia los ítems: Ambiente familiar, Mejor precio, Ambiente personalizado y Seguridad, ratifican que estos predeterminan la preferencia del cliente. Similar estudio confirma que en la preferencia del cliente, hacia un centro u otro, incide de forma directa el nivel adquisitivo, al respecto Haro-González, Pérez-Ordas, Leyton-Román, Caamaño-Guadix \& Nuviala (2018), demuestran en su investigación que existen dos grupos de usuarios de servicios deportivos y de fitness, uno con niveles altos de motivación autodeterminada, conformado por usuarios de centros exclusivos y un segundo grupo con niveles bajos de motivación autodeterminada, que asisten por lo regular a centros mixtos.

El modelo de regresión lineal muestra que los criterios arrojados reflejan una relación entre la calidad del servicio con la eficiencia y el ambiente laboral, cuando en el análisis factorial fueron incluidas todas las variables, esto puede ser un elemento denotativo del desconocimiento que sobre la actividad física y deporte poseen los actores de este tipo de emprendimientos.

En este punto es posible aseverar que el desarrollo de los emprendimientos están condicionados a varios factores dentro de los cuales se encuentra la segmentación del mercado, la forma de promoción de la información y la percepción del cliente en relación al servicio deportivo, estos ítems repercuten de forma significativa en la comercialización acertada del emprendimiento deportivo, por tanto la información del estado de estos indicadores, de acuerdo a varios autores, permiten el posicionamiento en el mercado (Funk, Filo, Beaton \& Pritchard, 2009; Sicilia, Águila, Muyor, Orta \& Moreno, 2009; Nuviala, et al., 2014; Sánchez-Alcaraz, \& Martínez, 2016; Nuviala, et al., 2017).

\section{Conclusiones}

A pesar de sus contribuciones, este estudio no está exento de limitaciones a tener en cuenta para la investigación futura. En primer lugar, se evidencia que desde las ciencias aplicadas al deporte se han hecho limitadas investigaciones con respecto a los emprendimientos deportivos (Abadia, et al., 2016). La profesión constituyó una de las variables caracterizada, tanto en cliente como en emprendedores, mediante esta fue posible identificar posibles ofertas para grupos de personas que no pueden asistir regularmente, necesidades, motivaciones e intereses hacia la práctica del ejercicio físico.

Como futura investigación se propone el diseño del modelo teórico de emprendimiento deportivo que cambie la realidad encontrada en la subregión Sabana y se extienda a las restantes región es del Departamento de Sucre, Mojana y Montes de María, que interrelacione los componentes validados en tres fases: básica, desarrolladora y resultante. El modelo corregirá las deficiencias detectadas en diagnóstico y otras ausentes en el estudio: Calidad de vida, Estilos de vida saludables e Inclusión social (Lamoneda-Prieto, \& Huertas-Delgado, 2017; FernándezVillarino, et. al., 2017; Leyton Román, García Matador, Fuentes García, \& Jiménez Castuera, 2018). Estas variables son relevantes según las condiciones económicas y sociales de la región estudiada. 


\section{Referencias}

Abadia, S. (2014). Las carreras populares en la Barcelona de la transición política (1975-1982). RICYDE. Revista internacional de ciencias del deporte, 36(10), 156-172.

Abadia, S., Medina, F.X., Sánchez, R., Sánchez, J., Bantulà, J., \& Morejón, S. (2016). Empresa, deporte y solidaridad: el caso de Oxfam Intermón Trailwalker RICYDE. Revista internacional de ciencias del deporte, 44(12), 164-181.

Acosta, R. (2012). Iniciación y Formación Deportiva: Una Reflexión Siempre Oportuna. Revista U.D.C.A Actualidad \& Divulgación Cientifica, (15), 57 65.

Alvariñas-Villaverde, M., López-Villar, C., Fernández-Villarino, M.A., \& AlvarezEsteban, R. (2017). Masculine, feminine and neutral sports: Extracurricular sport modalities in practice. Journal of Human Sport and Exercise, 12(4), 1278-1288. Recuperado de doi:https://doi.org/10.14198/jhse.2017.124.14

Arias, F. G (2017). Economía del Deporte: Estado de la cuestión y casos prácticos. Caracas: Editorial Episteme, C.A.

Ascenso-Campos, Francisco J., Da Costa-Simões, Vera A. \& Alves-Franco, Susana C. (2017). Qualidade do comportamento do instrutor em atividades de grupo de fitness. Revista Iberoamericana de Psicología del Ejercicio y el Deporte. 12(2), 249-259.

Barba-Sánchez, V., \& Atienza-Sahuquillo, C. (2018). Entrepreneurial intention among engineering students: The role of entrepreneurship education. European Research on Management and Business Economics, (24), 53-61.

Bojica, A., Fuentes, M., \& Ruiz, M. (2012). La adquisición de conocimiento a través de relaciones interorganizativas y la orientación emprendedora: el pape mediador del capital social de segundo orden. Cuadernos de Economía y Dirección de la Empresa, (15), 141-153.

Castro-López, R., Cachón, J., Valdivia-Moral, P. \& Zagalaz, M. L. (2015). Estudio descriptivo de trastornos de la conducta alimentaria y autoconcepto en usuarios de gimnasios. Revista Iberoamericana de Psicología del Ejercicio y el Deporte, 10 (2), 251-258

Dancey, C., \& Reidy, J. (2006). Estadísticas sin Matemáticas a la Psicología: Usar SPSS para Windows. Porto Alegre: Artmed. Revista Psicología, 8(1), 76-89.

Espejo, J. L., \& Ezquerra, S. (2002). Elaboración de un modelo de impacto del fútbol profesional en la economía española. Madrid: Editorial ESIC.

Fernández-Villarino M., González-Valeiro, M., Toja-Reboredo, B. \& Carreiro da Costa, F. (2017). Valoración de la escuela y la Educación Física y su relación con la práctica de actividad física de los escolares. Retos, 31(1), 312-315.

Fischer, L., \& Navarro, E. (1999). Introducción a la Investigación de Mercados. 2da edición Ed. McGraw Hill.

Formichella, M. M. (2004). El concepto de emprendimiento y su relación con la educación, el empleo y el desarrollo local. Instituto Nacional de Tecnología Agropecuaria. INTA. Rivadavia 1439 (1033), Buenos Aires, Argentina.

Funk, D. C., Filo, K., Beaton, A. A., \& Pritchard, M. (2009). Measuring the motives of sport event attendance: Bridging the academicpractitioner divide to understanding behaviour. Sport Marketing Quartely, 18(3), 126-138.

García, F. G. \& Pires, V. F. (2010). Fidelización de usuarios mayores en centros de fitness: Gestión de clientes por programas de actividad física. Retos. Nuevas tendencias en Educación Física, Deporte y Recreación, (17), 103-106

García, J., Bernal, A., Lara, A., \& Galán, P. (2013). La calidad percibida de servicio y su influencia en la fidelidad de usuarios mayores en centros de fitness públicos. Escritos de Psicología, 6(2), 32-47.

Gil-Madrona, P., Pita-Lozano, A., Díaz-Suárez, A., \& López-Sánchez, G. F. (2017). Validación del cuestionario: «Perception of competence in middle school PE» al contexto español. RICYDE. Revista internacional de ciencias del deporte, 48(13), 172-187.

González-Montesinos, J. L., \& Fernández-Santos, J. R. (2012). Origen y evolución de las patentes y marcas en biomecánica deportiva. Revista internacional de ciencias del deporte, 30(8), 276-304

Guillen, P. L., Arma, C. N., \& Formoso, M. A. (2018). La inclusión laboral de la mujer en el proceso de crecimiento empresarial. Universidad y Sociedad, 10(2), 128-138.

Guillen, P. L., Martinez, G. L., \& Montano, R. F. (2018). La responsabilidad social en el Marketing desportivo. Universo y Sociedad 10(2), 175-180.

Haro-González, M., Pérez-Ordas, R., Leyton-Román, M., Caamaño-Guadix, A., \& Nuviala, A. (2018). Grupos de usuarias del mercado deportivo femenino. Retos, (34), 123-127.

Herrera, B. (2014). Emprendimiento. Algunos conceptos de emprendimiento. Blogspot.com. Obtenido de blogspot.com: http:// emprendimiento110.blogspot.com.co/

Holcombe, Randall G. (1998). Entrepreneurship and Economic Growth. Quarterly Journal of Austrian Economics, 1(2), 45-62.

Huertas, M., Valantine, I., \& Crespo, H. J. (2014). La investigación sobre emprendimiento en el ámbito deportivo. Revisión de los documentos publicados en la WOS. Journal of Sports Economics \& Management, 4(1), 55-66.

Kelley, D., Singer, S., \& Herrington, M. (2016). Global Report 2015-2016. Editor Global Entrepreneurship Monitor (GEM).

Lamoneda-Prieto, J.r, \& Huertas-Delgado, F. J. (2017). Análisis de la práctica deportiva-recreativa a través de un programa de promoción en el recreo en función del sexo en adolescentes españoles. Retos, 32 (2), 25-29.

Leyton Román, M., García Matador, J., Fuentes García, J. P. \& Jiménez Castuera, R. (2018). Análisis de variables motivacionales y de estilos de vida saludables en practicantes de ejercicio físico en centros deportivos en función del género. Retos, 34 (2).

Martínez del Castillo, J., Jiménez-Beatty, J.E., Santacruz, J.A., Martín, M., \& Rivero, A. (2011) La recomendación médica y el tipo de demanda de actividad física en las personas mayores de la provincia de Guadalajara. Revista internacional de ciencias del deporte, 23(7), 92-102.

Martínez, J. A. (2015). David Falk: Algunas cuestiones abiertas para el marketing deportivo. RICYDE. Revista internacional de ciencias del deporte, 40(11), 186-191.

Méndez, L. R. (2015). Centro de Emprendimiento e Innovación. Una estrategia de desarrollo institucional. ENTORNOS, 28(2), 129.

Morales, V., \& Gálvez, P. (2011). La percepción del usuario en la evaluación de la calidad de los servicios municipales deportivos. Cuadernos de Psicología del Deporte, 2 (2), 147-154.

Mora-Rodríguez, R. (2009). Fisiología del deporte y el ejercicio: prácticas de campo. Buenos Aires: Editorial Médica Panamericana

Moreno-Murcia, J. A., Cervelló-Gimeno, E., Martínez-Galindo, C., \& Moreno, R. (2013). Validación de la Escala de Creencias Implícitas de habilidad(CNAAQ2) al contexto español. Diferencias según la práctica físico-deportiva. RICYDE. Revista internacional de ciencias del deporte, 32(9), 100-113.

Murillo, B., J., J.A., García-González, L., Albarca-Sos, A., \& Zaragoza, J. (2014). Influencia del género y de los contenidos sobre la actividad física y la percepción de competencia en Educación Física. RICYDE. Revista internacional de ciencias del deporte, 36(10), 131-143.

Nuviala, A., Tamayo-Fajardo, J. A., Ruiz-Alejos, G. C., Nuviala, N. R., \& Dalmau, T. J. M. (2017). Adaptación y validación del cuestionario Epod 2.1 a usuarios libres de centros deportivos. Retos, (31), 40-45.

Nuviala, N. R., Teva-Villén, M. R., Pérez-Ordás, R., Grao-Cruces, A., Tamayo, F. J. A., \& Nuviala, N. A. (2014). Segmentación de usuarios de servicios deportivos. Retos. Nuevas tendencias en Educación Física, Deporte y Recreación, (25), 90-94

Puigarnau, S., Camerino, O., Castañer, M., Prat, Q., \& Anguera, M.T. (2016). El apoyo a la autonomía en practicantes de centros deportivos y de fitness para aumentar su motivación. RICYDE. Revista internacional de ciencias del deporte, 43(12), 48-64

Pullés, D., Gutiérrez, L., \& Llorens, J. (2013). Transactive memory system and TQM: exploring knowledge capacities. Industrial Management y Data Systems, (113), 294 - 318.

Ramírez, C., Lorenzo, A., \& López, C. (2016). Revisión de los Instrumentos de Medida de la Calidad en los Servicios Deportivos. Kronos. 16(1). Recuperado de: https://g-se.com/revision-de-los-instrumentos-de-medida-de-la-calidad-enlos-servicios-deportivos-2299-sa-M59681b60caa6e

Ratten, V. (2012). Sport entrepreneurship: challenges and directions for future research. International Journal of Entrepreneurial Venturing, 4(1), 65-76.

Ratten, V., \& Ratten, H. (2011). International sport marketing: practical and future research implications. Journal of Business y Industrial Marketing, 26(8), 614620

Rial, J.; Varela, J., \& Rial, A.; Real, E. (2010). Modelización y medida de la Calidad Percibida en centros Modelización y medida de la Calidad Percibida en centros deportivos: la escala QSport-10. Deportivos: la escala QSport-10. Revista Internacional de Ciencias del Deporte. 18(6), 57-73

Rodríguez,A. (2009). Nuevas perspectivas para entender el emprendimiento empresarial. Revista científica Pensamiento y Gestión, (26), 94-119.

Sánchez-Alcaraz, M. B., \& Martínez, M. R. (2016). Perfil, Preferencias y Satisfacción de los Clientes de Centros de Fitness Low-Cost. Revista de Educación Física, 34(3), 1-7.

Sevil, J., Abós, A., Julian, J. A., Murillo, B., \& García-González, L. (2015). Género y motivación situacional en Educación Física: claves para el desarrollo de estrategias de intervención. RICYDE. Revista internacional de ciencias del deporte, 41(11), 281-296.

Sicilia, A., Águila, C., Muyor, J. M., Orta, A., \& Moreno, J.A. (2009). Perfiles motivacionales de los usuarios en centros deportivos municipales. Anales de psicología, 25(1), 160-168.

Stevenson, H., \& Jarillo, C. (1983). A Paradigm of Entrepreneurship: Entrepreneurial Management. Reviewed work(s): Source. Strategic Management Journal, (11), 17-27.

Suárez, L., \& Vásquez, C. (2015). Evolución del Concepto de Emprendedor: de Cantillón a Freire. REDIP. UNEXPO. Revista Digital de Investigación y Postgrado, 5(3), 882-894.

Tuero, P. C., \& Marquez, S. (2010). Actividad física y salud. Estrategias de medición y valoración de la actividad física. Editorial: FUNIBER, Díaz de Santos, (1), 51-68.

Urteaga, E. (2013). El pensamiento de Norbert Elias: proceso de civilización y configuración social Barataria. Revista Castellano- Manchega de Ciencias Sociales, (16), 15-31

Xavier, W., Martins, G, \& Lima, A. (2008). Fortalecendo empreendimentos em TI: qual a contribuição das Incubadoras. Gestão da Tecnologia e Sistemas de Informação, 5(3), 433-452. 